\title{
Frühe aggressive intravenöse Volumentherapie
}

Hintergrund: Die akute Pankreatitis stellt in der Gastroenterologie einen der häufigsten Gründe für eine stationäre Behandlung über die Notaufnahme dar. Eine pharmakologische Therapie, die den Verlauf der akuten Pankreatitis positiv beeinflussen kann, existiert nicht, daher bleibt die Behandlung rein symptomatisch. Im klinischen Alltag wird trotz fehlender Belege durch randomisierte Studien die frühzeitige, intensivierte intravenöse Flüssigkeitsgabe bei der akuten Pankreatitis empfohlen.

Patienten und Methoden: In dieser randomisierten Studie wurde eine aggressive Hydrierung $(20 \mathrm{ml} / \mathrm{kg}$ Bolus gefolgt von $3 \mathrm{ml} /$ $\mathrm{kg} / \mathrm{h})$ mit einer standardisierten Flüssigkeitsgabe $(10 \mathrm{ml} / \mathrm{kg}$ Bolus gefolgt von $1,5 \mathrm{ml} / \mathrm{kg} / \mathrm{h}$ ) mit Ringer-Laktat-Lösung (RL) in der frühen Behandlung der milden akuten Pankreatitis verglichen. 60 Patienten mit einer akuten Pankreatitis ohne Zeichen eines „Systemic Inflammatory Response Syndrome“ (SIRS) und ohne Organversagen wurden eingeschlossen (Atlanta-Klassifikation [1]). Folgende Parameter wurden alle zwölf Stunden erhoben: Hämatokrit, Serumharnstoff, Serumkreatinin. Kam es zu einem Anstieg einer dieser Parameter in einer der beiden Gruppen, erhielten die Patienten einen Bolus ( $20 \mathrm{ml} / \mathrm{kg}$ gefolgt von $3 \mathrm{ml} / \mathrm{kg} / \mathrm{h})$. Bei klinischer (Schmerzen) und laborchemischer Verbesserung wurde die Volumengabe auf $1,5 \mathrm{ml} / \mathrm{kg} / \mathrm{h}$ reduziert und eine orale flüssige Ernährung begonnen. Der primäre Endpunkt war die klinische Verbesserung innerhalb von 36 Stunden (definiert als Kombination aus Absinken des Hämatokrits, des Serumharnstoffs und des Kreatinins, Besserung der Schmerzen und Verträglichkeit der oralen Ernährung). Das mittlere Alter der Patienten betrug 45

Buxbaum JL et al. Early Aggressive Hydration Hastens Clinical Improvement in Mild Acute Pancreatitis. Am J Gastroenterol 2017; 112 (5): $797-803$ Jahre und bei 14 (23\%) Patienten bestanden Komorbiditäten, zehn (37\%) wurden als alkoholinduzierte akute Pankreatitis klassifiziert.

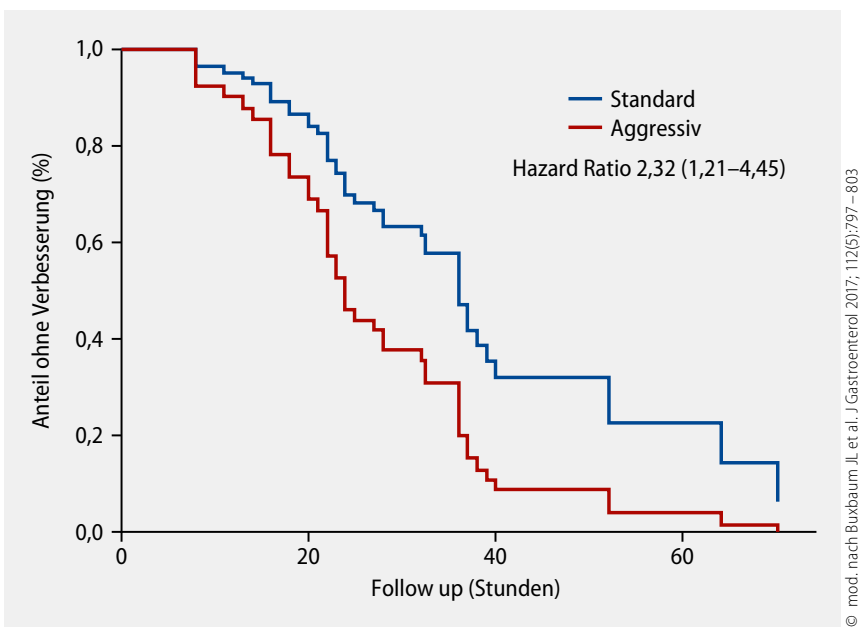

1 Klinische Verbesserung der aggressiven versus Standardflüssigkeits-

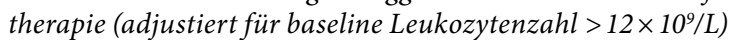

Ergebnisse: Ein signifikant höherer Anteil der Patienten in der Gruppe aggressiver Flüssigkeitstherapie zeigte nach 36 Stunden eine klinische Verbesserung, als Patienten im Standardflüssigkeitsarm: $70 \%$ vs. $42 \%(\mathrm{p}=0,02)(\triangleright A b b .1)$; ein persistierendes SIRS trat in der Gruppe mit aggressiver Flüssigkeitsgabe seltener auf $(7,4 \%$ vs. $21,1 \%$; Odds Ratio [OR] $=0,12 ; 0,02-0,94)$. Gleiches galt für eine Hämokonzentration $(11,1 \%$ vs. $36,4 \%$, $\mathrm{OR}=0,08,0,01-0,49)$. Bei keinem der Patienten in beiden Gruppen konnten Zeichen einer Volumenüberladung gesehen werden.

Schlussfolgerung: Die frühe aggressive Flüssigkeitsgabe mit RL trägt zu einer Beschleunigung der klinischen Verbesserung bei milder akuter Pankreatitis bei.

\section{- Kommentar von Ulrike von Arnim, Magdeburg}

\section{Klinische Besserung bei intensivierter Therapie mit Ringer-Laktat-Lösung}

\begin{abstract}
Diese klinisch relevante Arbeit kann zur Verbesserung des Managements bei milder akuter Pankreatitis beitragen. Das einfache und klare Design der Studie unterstreicht die Praktikabilität des früh-aggressiven Infusionsregimes und kann ein signifikant verbessertes klinisches Outcome nach 36 Stunden bei Patienten mit akuter Pankreatitis zeigen. Anzumerken ist, dass Patienten mit einer moderaten und schweren Pankreatitis nicht eingeschlossen wurden, dies, weil die frühe intensivierte Flüssigkeitstherapie in einigen Studien nachteilige Effekte auf den Verlauf der akuten Pankreatitis gehabt hatte. Bei einer milden akuten Pankreatitis stellt das in der Studie vorgeschlagene früh aggressive Infusionsmanagement eine leicht zu realisierende und im klinischen Management der akuten Pankreatitis erfolgreiche therapeutische Intervention dar.
\end{abstract}

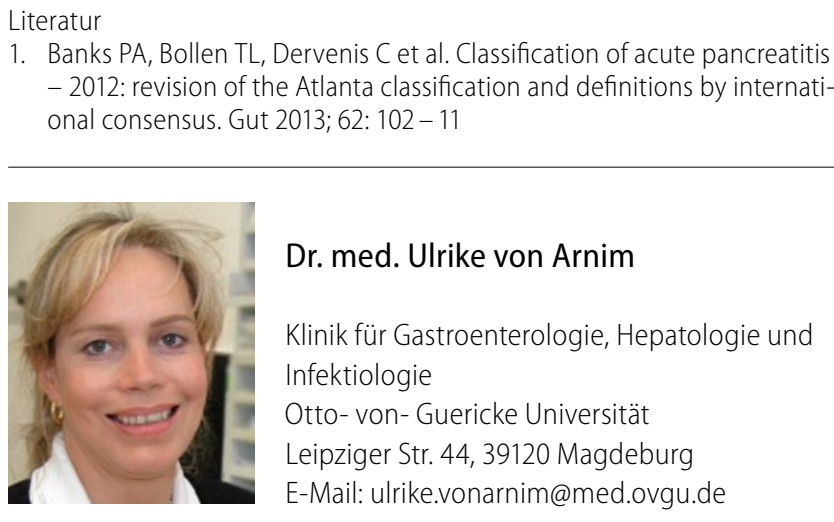

Literatur

Banks PA, Bollen TL, Dervenis C et al. Classification of acute pancreatitis - 2012: revision of the Atlanta classification and definitions by international consensus. Gut 2013; 62: 102 - 11

Dr. med. Ulrike von Arnim

Klinik für Gastroenterologie, Hepatologie und Infektiologie

Otto- von- Guericke Universität

E-Mail: ulrike.vonarnim@med.ovgu.de 